\title{
Effets antidiabétiques de l'extrait aqueux de Ageratum conyzoïdes (Astéraceae) chez les rats rendus diabétiques par pancréatectomie partielle et évaluation de leurs paramètres hématologiques
}

\author{
Effozougba Jean-Benoît ONSIYOR ${ }^{1^{*}}$, Nicaise Akaffou AKAFFOU ${ }^{2}$, \\ Ouga Stanislas ZAHOUI $^{1}$ et Flavien TRAORE ${ }^{1}$ \\ ${ }^{1}$ Laboratoire de Physiologie Animale, U.F.R. Biosciences, Université Félix Houphouët Boigny, 22 B.P. 582 \\ Abidjan 22, Côte d'Ivoire. \\ ${ }^{2}$ Bureau Ivoirien pour la Représentation et le Conseil Vétérinaire (BIRCOVET) 23 BP 3657 Abidjan 23, \\ Côte d'Ivoire. \\ *Auteur correspondant ; E-mail : effozougba@gmail.com; Tel: (+225) 87876357/05137813
}

\section{RESUME}

L'objectif de ce travail a été de justifier l'utilisation traditionnelle de Ageratum conyzö̈des dans le traitement du diabète sucré. Pour ce faire, quatre lots de six rats ont été constitués. Les animaux de trois lots ont été rendus diabétiques par pancréatectomie partielle; les rats des lots 3 et 4 ont reçu respectivement des doses quotidiennes de glibenclamide à $10 \mathrm{mg} / \mathrm{Kg}$ p.c. et d'extrait aqueux de Ageratum conyzoïdes (EAqAc) à 5000 $\mathrm{mg} / \mathrm{Kg}$ p.c. pendant 28 jours. La glycémie des rats a été mesurée par intervalle régulier de 7 jours. A la fin de l'expérimentation, les rats ont été mis à jeun et anesthésiés à l'éther di éthylique, puis leur sang a été prélevé au niveau du sinus orbital sans abimer l'œil à l'aide d'une pipette pasteur, afin de déterminer leurs paramètres hématologiques. Les résultats ont montré que, l'EAqAc et le glibenclamide, baissent significativement ( $<<0,001$ ) la glycémie respectivement de $74,39 \%$ et de $75,01 \%$ chez les rats traités, comparativement aux animaux témoins. Les paramètres hématologiques des rats diabétiques traités à l'EAqAc, ne varient pas significativement $(\mathrm{p}>0,05)$ par rapport à ceux des rats normo-glycémiques. Cet extrait pourrait agir comme le glibenclamide et serait un régulateur des paramètres hématologique.

(C) 2019 International Formulae Group. All rights reserved

Mots clés : Diabète, Hématologique, Glibenclamide, Glycémie.

\section{Antidiabetic effects of aqueous extract of Ageratum conyzoides (Astéraceae) in rats made diabetic by partial pancreatectomy and evaluation of their haematological parameters}

\begin{abstract}
The aim of this work was to justify the traditional use of Ageratum conyzoides in the treatment of diabetes mellitus. To do this, four lots of six rats were formed. Animals from three lots were made diabetic by partial pancreatectomy; the rats in batches 3 and 4 received daily doses of $10 \mathrm{mg} / \mathrm{kg}$ of glibenclamide and aqueous extract of Ageratum conyzoides (EAqAc) at $5000 \mathrm{mg} / \mathrm{kg}$ pw. for 28 days. The blood glucose levels of the rats were measured at a regular interval of 7 days. . At the end of the experiment, the rats were fasted and
\end{abstract}


anesthetized with diethyl ether, then their blood was taken from the orbital sinus without damaging the eye using a Pasteur pipette, to determine their hematological parameters. The results showed that EAqAc and glibenclamide significantly decreased $(\mathrm{p}<0.001)$ blood glucose by $74.39 \%$ and $75.01 \%$, respectively, in treated rats compared to control animals. The haematological parameters of the diabetics rats treated with EAqAc do not vary significantly ( $p>0.05$ ) compared to those of normoglycemic rats. This extract could act as glibenclamide and be a regulator of haematological parameters.

(C) 2019 International Formulae Group. All rights reserved

Keywords : Diabetes, Haematologic, Glibenclamide, Blood Glucose.

\section{INTRODUCTION}

Le diabète est une maladie métabolique qui est devenue un problème de santé publique (FID, 2014). Selon les estimations, 5\% de la population mondiale souffraient de diabète en 2014 et plus de 41 million de diabétiques sont attendus en 2035 (FID, 2014). Selon l'OMS, 5,6\% des hommes et $4,4 \%$ des femmes de la population ivoirienne souffrent de diabète (OMS, 2016). Le traitement du diabète reste depuis longtemps restreint aux changements de régime alimentaire, à l'injection d'insuline et à la prise d'antidiabétiques oraux (Gbekley et al., 2015). Le coût excessif de ces agents antidiabétiques et l'insuffisance des infrastructures médicales, associés au manque de personnel soignant en Afrique amènent les populations à s'orienter vers la médecine traditionnelle. Les plantes médicinales constituent un potentiel médical accessible, disponible et à moindre coût (Okigbo et Omodamiro, 2006). Ces plantes constituent une ressource intarissable qui fournit la majorité des principes actifs des produits pharmaceutiques. Cependant une multitude de ces plantes médicinales utilisées n'ont pas de données scientifiques sur leurs efficacités et leurs innocuités (Fleurentin, 2012). En effet pour une utilisation rationnelle de ces plantes, des travaux doivent être réalisés afin de déterminer les possibles effets néfastes induits par l'utilisation de celles-ci et qui pourraient entrainer d'autres complications dans le traitement des pathologies (Azzi et al., 2012). C'est dans cette perspective que nous avons entrepris d'étudier les effets de l'extrait aqueux de Ageratum conyzoïdes (Astéraceae), une plante de la pharmacopée ivoirienne, utilisée dans le traitement du diabète.
L'objectif de ce travail était de justifier l'utilisation traditionnelle de Ageratum conyzö̈des, dans le traitement du diabète sucré, par l'étude des effets de son extrait aqueux sur des rats rendus diabétiques par pancréatectomie partielle et l'évaluation de leurs paramètres hématologiques.

\section{MATERIEL ET METHODES \\ Matériel végétal}

Le matériel végétal utilisé est constitué de la plante entière de Ageratum conyzö̈des Linn. (Asteraceae), achetée chez des herboristes au marché de Yopougon (une commune de la ville d'Abidjan). Cette plante a été identifiée par le professeur Guédé Zirihi du laboratoire de Botanique de l'Université Félix Houphouët Boigny-Abidjan. Elle est répertoriée sous le numéro 12276 d'Agboville de l'herbier du Centre National de Floristique de l'Université Félix Houphouët BoignyAbidjan.

\section{Matériel animal}

Des rats de l'espèce Rattus norvegicus de souche Wistar, pesant entre 180 et 213 grammes ont été utilisés. Ces rats sont nourris essentiellement aux graminées et vivent à la température ambiante moyenne de $28 \pm 4{ }^{\circ} \mathrm{C}$, dans une atmosphère contenant $65 \%$ d'humidité, la photopériode est de 12 heures/24 heures.

\section{Produits chimiques}

\section{Le CHLOROFORME ${ }^{\circledR} \quad$ (MERCK, $^{\circ}$}

ALLEMAGNE) a été utilisé pour anesthésier les animaux. La teinture d'iode $10 \%$,

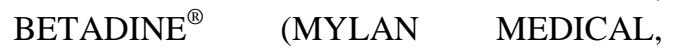
FRANCE) et l'alcool $90^{\circ}$ ont servi à la stérilisation et au pansement des zones à 
inciser. L'usage d'ALUSPRAY ${ }^{\circledR}$ (VETOQUINOL, FRANCE) a permis de cicatriser les plaies. Le CLAMOXYL ${ }^{\circledR}$ (GLAXOSMITHKLINE, ROYAUME-UNI) a servi à prévenir les infections. Comme antalgique nous avons utilisé

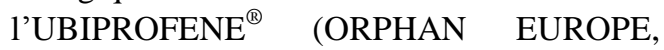
FRANCE). Le Glibenclamide (GLIDIABET ${ }^{\circledR}$ (FERRER INTERNATIONAL SA, Espagne) a servi d'hypoglycémiant de référence et l'EDTA (Ethylène Diamine Tétra Acétique) a été utilisé pour éviter la coagulation du sang prélevé par capillarité au niveau du sinus orbital.

\section{Préparation de l'extrait aqueux de Ageratum conyzoïdes}

La plante entière a été séchée à température ambiante $\left(28 \pm 4{ }^{\circ} \mathrm{C}\right)$. Cinquante grammes $(50 \mathrm{~g})$ de poudre séchées de Ageratum conyzoïdes ont été mixés pendant 24 heures dans 2 litres d'eau distillée sous agitation magnétique. La solution obtenue a été filtrée sur du coton hydrophile et du papier WATTMAN (3 mn). Le filtrat obtenu a été évaporé sous vide à $70 \quad{ }^{\circ} \mathrm{C}$ grâce à un évaporateur Rotavapor de type Bucchi. La pâte obtenue a été congelée puis lyophilisée. On a ainsi obtenu une poudre soluble dans l'eau utilisée pour préparer les solutions tests.

\section{Induction du diabète par pancréatectomie partielle}

Le rat anesthésié par inhalation de chloroforme a été placé sur une planche à dissection et la partie abdominale a été aseptisée puis rasée à l'aide d'un bistouri avant d'être à nouveau aseptisée. Une laparotomie médiane de la zone située à équidistance entre la pointe du sternum et la zone ombilicale a été effectuée, afin d'isoler le pancréas et de le broyer partiellement de manière irréversible. La cavité abdominale a été refermée par des points de sutures. La plaie a été nettoyée avec un tampon de teinture d'iode $10 \%$ suivi de la pulvérisation d'un cicatrisant d'aluminium micronisé (ALUSPRAY ${ }^{\circledR}$ ). On a effectué deux injections: Amoxicilline $(0,2 \mathrm{ml})$ et AINS $(0,2 \mathrm{ml})$ par voie intramusculaire (IM) au niveau des muscles de la masse commune. Ce traitement a été renouvelé pendant trois jours consécutifs. La glycémie de ces animaux opérés était contrôlée chaque semaine.

Au bout de six (06) semaines, tout rat dont la glycémie était supérieure à $300 \mathrm{mg} / \mathrm{dl}$ était considéré comme rat diabétique et était utilisé pour les expérimentations.

Evaluation des effets de l'extrait aqueux de Ageratum conyzoïdes (EAqAc) sur la glycémie chez les rats rendus diabètes par pancréatectomie partielle

Vingt-quatre (24) rats Wistar repartis en quatre (04) lots de six (06) rats chacun ont été utilisés.

Le lot 1 était composé de rats normaux non diabétiques, qui sont les témoins normoglycémiques (TNG), recevant deux (02) $\mathrm{ml}$ d'eau distillée durant toute la durée de l'expérience. Le lot 2 était composé de rats diabétiques, qui sont les témoins diabétiques (TD), traités avec deux (02) ml d'eau distillée durant toute l'expérience. Le lot 3 était composé de rats diabétiques qui recevaient une dose quotidienne de $10 \mathrm{mg} / \mathrm{kg}$ p.c. de Glibenclamide (Glib), la substance hypoglycémiante de référence (R-Glib). Le lot 4 comprenait des rats diabétiques qui étaient gavés quotidiennement avec deux (02) $\mathrm{ml}$ de $5000 \mathrm{mg} / \mathrm{kg}$ p.c. de l'EAqAc pendant la durée des expérimentations (R-EAqAc). Cette dose de $5000 \mathrm{mg} / \mathrm{kg}$ p.c. a été choisie après une étude toxicologique.

Les mesures de la glycémie ont été faites pour chaque lot à des intervalles de temps régulier de sept (7) jours jusqu'au vingt-huitième $\left(28^{\text {ème }}\right)$ jour de traitement grâce au glucomètre de marque On Call EZ II de Acon Diabetes Care International. Les variations de la glycémie ont été calculées grâce à la formule suivante :

$\mathrm{V}_{\mathrm{G}}=\frac{\mathrm{Gx} \times 100}{\mathrm{G} 1}-100$

$\mathrm{V}_{\mathrm{G}}=$ Variation de la glycémie, $\mathrm{Gx}=$ Valeur de la glycémie au jour $\mathrm{x}, \mathrm{x}=$ Jour du prélèvement, G1= Valeur de la glycémie au jour 1 


\section{Effets de l'extrait aqueux de Ageratum conyzoüdes (EAqAc) sur les paramètres hématologiques}

Pour cette étude, trois (03) lots parmi les 4 lots utilisés ont été choisis pour l'étude de la glycémie. Il s'agit du lot 1 de rats témoins normo-glycémiques (TNG), du lot 2 de rats témoins diabétiques (TD) et du lot 4 de rats diabétiques traités avec l'EAqAc à 5000 $\mathrm{mg} / \mathrm{kg}$ p.c. L'influence de cette dose d'EAqAc sur les paramètres hématologiques a été appréciée à la fin des vingt-huit (28) jours d'expérimentation. A la fin de l'expérimentation, les rats des trois (3) lots ont été mis à jeun et anesthésiés à l'éther diéthylique, puis le sang de ces animaux a été prélevé au niveau du sinus orbital sans abimer l'œil à l'aide d'une pipette pasteur et conservé avec un anticoagulant EDTA (Ethylène Diamine Tétra Acétique).

L'hémogramme a été réalisé directement par l'analyseur automatique Sysmex poch-100i (USA). Ainsi, les taux de globule rouge, de globule blanc, de volume globulaire moyen, de plaquettes sanguines, d'hémoglobine et d'hématocrite ont été déterminés.

\section{Analyses statistiques}

Le traitement statistique des données a été réalisé grâce au logiciel Graph PadInstat 3 (San Diégo, Californie USA). La différence statistique entre les moyennes a été réalisée grâce à l'analyse des variances (ANOVA), suivie du test de comparaison multiple de Tukey6Kramer, avec un seuil de significativité $\mathrm{P}<0,05$. Toutes les valeurs sont présentées sous la forme de moyenne \pm ESM (Erreur Standard sur la Moyenne).

\section{RESULTATS}

Effets de l'extrait aqueux de Ageratum conyzoïdes sur la glycémie chez les rats rendus diabétiques par pancréatectomie partielle

Les valeurs des différentes glycémies prélevées sont consignées dans le Tableau 1.

$\mathrm{La}$ dose de $5000 \mathrm{mg} / \mathrm{kg}$ p.c. de l'EAqAc entraine chez les rats rendus diabétiques par pancréatectomie partielle, une baisse de la glycémie après sept (07) jours de traitement. Cette glycémie passe de $366,4 \pm 4,02$ à $335,9 \pm 4,7 \mathrm{mg} / \mathrm{dl}$ soit une diminution significative $(\mathrm{p}<0,05)$ de $8,32 \%$ par rapport à la glycémie de départ. Cette glycémie passe à $289,9 \pm 6,1 \mathrm{mg} / \mathrm{dl}$ après quatorze (14) jours de traitement soit une réduction significative ( $\mathrm{p}<0,001)$ de $20,87 \%$ comparé à la glycémie de départ. Après 21 jours de traitement, la glycémie est de $200,78 \pm 6,89 \mathrm{mg} / \mathrm{dl}$ soit une baisse significative ( $\mathrm{p}<0,001$ ) de $45,20 \%$ par rapport à la glycémie de départ.

La réduction maximale entrainée par cette dose est observée après vingt-huit (28) jours avec une baisse significative $(\mathrm{p}<0,001)$ de $74,39 \%$, faisant passer la valeur de la glycémie de $366,4 \pm 4,02$ à $93,8 \pm 6,66 \mathrm{mg} / \mathrm{dl}$

La molécule de référence, le Glibenclamide à $10 \mathrm{mg} / \mathrm{kg}$ p.c. a diminué significativement $(\mathrm{p}<0,001)$ le niveau de l'hyperglycémie de $44,27 \%, 72,02 \%$ et $75,01 \%$ après respectivement 14,21 et 28 jours de traitement chez les rats diabétiques comparé à leur glycémie de départ. Chez les animaux témoins normo-glycémiques, la glycémie ne varie presque pas durant toute l'expérimentation. En revanche, la glycémie augmente significativement (p <0,001), comparée à la glycémie de départ, chez les rats témoins diabétiques. Elle passe de 325,9 à $398,98 \mathrm{mg} / \mathrm{dl}$ au jour vingt-huit (28).

Effets de l'extrait aqueux de Ageratum conyzoïdes (EAqAc) sur les paramètres hématologiques chez les rats rendus diabétiques par pancréatectomie partielle

Le Tableau 2 représente les effets de l'extrait aqueux de Ageratum conyzoïdes sur les paramètres hématologiques des rats après vingt-huit (28) jours d'expérimentation.

Les paramètres hématologiques des rats traités avec $5000 \mathrm{mg} / \mathrm{kg}$ p.c. pendant vingt-huit (28) jours ne varient pas significativement $(p>0,05)$ de ceux des rats témoins normo-glycémiques. En revanche, les taux de globule rouge, du volume globulaire moyen et d'hématocrite des rats témoins diabétiques non traités baissent significativement $(\mathrm{p}<0,05)$ par rapport aux rats témoins normo-glycémiques. Cette baisse est plus significative $(\mathrm{p}<0,01)$ pour le taux d'hémoglobine. Le taux de globule blanc subit une augmentation significative $(\mathrm{p}<0,05) \mathrm{chez}$ les rats témoins diabétiques par rapport aux rats témoins normo-glycémiques. 
Tableau 1 : Variation de la glycémie des lots de rats durant 28 jours.

\begin{tabular}{|c|c|c|c|c|c|c|}
\hline \multirow{2}{*}{$\begin{array}{l}\text { Modèles } \\
\text { d'animaux }\end{array}$} & & \multicolumn{5}{|l|}{ jours } \\
\hline & & $\mathbf{J}+\mathbf{1}$ & $\mathrm{J}+7$ & $\mathbf{J + 1 4}$ & $\mathrm{J}+21$ & $\mathrm{~J}+28$ \\
\hline \multirow{2}{*}{$\begin{array}{l}\text { TNG } \\
\mathrm{n}=6\end{array}$} & Glycémie (mg/dl) & $83,65 \pm 4,79$ & $80,79 \pm 5,6$ & $82,89 \pm 4,98$ & $82,6 \pm 4,89$ & $81,16 \pm 5$ \\
\hline & Var. glycémie (\%) & & $-3,41 \%$ & $-0,90 \%$ & $-1,25 \%$ & $-2,97 \%$ \\
\hline \multirow{2}{*}{$\begin{array}{l}\mathrm{TD} \\
\mathrm{n}=6\end{array}$} & Glycémie en mg/dl & $325,9 \pm 5,4$ & $330,9 \pm 4,89$ & $382,6 \pm 5,8$ & $388,9 \pm 5,45$ & $398,98 \pm 6,12$ \\
\hline & Var. glycémie (\%) & & $+1,53 \%$ & $+17,39 \%$ *** & $+19,33 \% * * *$ & $+22,42 \% * * *$ \\
\hline \multirow{2}{*}{$\begin{array}{l}\text { R-Glib } \\
\mathrm{n}=6\end{array}$} & Glycémie en mg/dl & $352,1 \pm 8,01$ & $330,2 \pm 8,3$ & $196,2 \pm 2,9$ & $98,5 \pm 5,4$ & $88 \pm 4,1$ \\
\hline & Var. glycémie (\%) & & $-6,21 \%$ & $-44,27 \% * * *$ & $-72,02 \% * * *$ & $-75,01 \% * * *$ \\
\hline \multirow{2}{*}{$\begin{array}{l}\text { R-EAqAc } \\
5000 \quad \mathrm{mg} / \mathrm{kg} \\
\text { p.c. } \\
\mathrm{n}=6\end{array}$} & Glycémie en mg/dl & $366,4 \pm 4,02$ & $335,9 \pm 4,7$ & $289,9 \pm 6,1$ & $200,78 \pm 6,89$ & $93,8 \pm 6,66$ \\
\hline & Var. glycémie (\%) & & $-8,32 \% *$ & $-20,87 \% * * *$ & $-45,20 \% * * *$ & $-74,39 \% * * *$ \\
\hline
\end{tabular}

$T N G=$ Témoins Normo-glycémiques $; T D=$ Témoins Diabétiques $; R$-Glib=Rats traités avec le Glibenclamide $; R$-EAqAc $=$ Rats traités avec l'extrait aqueux d'Ageratum conyzö̈des ; (-): Diminution, (+) : Augmentation, Var.=Variation; *= différence significative à $p<0,05 ; * *=$ différence significative à $p<0,01 ; * * *=$ différence significative à $p<0,001$.

Tableau 2: Effets de l'extrait aqueux d'Ageratum conyzoïdes sur la variation des paramètres hématologiques des rats après 28 jours de traitement.

\begin{tabular}{|c|c|c|c|c|}
\hline Paramètres hématologiques & TNG & TD & $\begin{array}{l}\text { R-EAqAc } \\
\text { mg/kg p.c. }\end{array}$ & 5000 \\
\hline Taux de Globule Rouge $\left(10^{6} / \mu \mathrm{l}\right)$ & $7,5 \pm 0,3$ & $4,8 \pm 0,6^{*}$ & $6,6 \pm 0,5$ & \\
\hline Taux de Globule blanc $\left(10^{3} / \mu \mathrm{l}\right)$ & $9,8 \pm 0,8$ & $13,7 \pm 0,9 *$ & $10 \pm 0,6$ & \\
\hline Volume Globulaire Moyen (fl) & $58 \pm 1,2$ & $48 \pm 1,8 *$ & $54 \pm 2,1$ & \\
\hline Taux de Plaquettes sanguines $\left(10^{3} / \mu \mathrm{l}\right)$ & $926 \pm 4,9$ & $745 \pm 3,2$ & $918 \pm 4,7$ & \\
\hline Taux d'Hémoglobine (g/dl) & $14,1 \pm 0,4$ & $9,2 \pm 0,7 * *$ & $12 \pm 0,9$ & \\
\hline Taux d'Hématocrite (\%) & $41,9 \pm 0,9$ & $33,8 \pm 2 *$ & $38 \pm 0,6$ & \\
\hline
\end{tabular}

$T N G=$ Témoins Normo-glycémiques $; T D=$ Témoins Diabétiques $; R$-EAqAc=Rats traités avec l'extrait aqueux de Ageratum conyzö̈des; $*$ différence significative à $p<0,05 ; * *=$ différence significative à $p<0,01 ; * * *=$ différence significative à $p<0,001$. 


\section{DISCUSSION}

L'administration de l'extrait aqueux de Ageratum conyzoïdes à $5000 \mathrm{mg} / \mathrm{kg}$ p.c. et du Glibenclamide à $10 \mathrm{mg} / \mathrm{kg}$ p.c., induit respectivement une baisse significative $(p<0,001)$ de $74,39 \%$ et de $75,01 \%$ de la glycémie chez les lots de rats diabétiques traités comparativement aux lots témoins chez lesquels il n'y avait pas de variation significative (cas des TNG) et une augmentation de la glycémie (cas des TD).

Cet effet observé est similaire aux effets de plusieurs plantes de la pharmacopée traditionnelle telles que Momordica charantia L. (Cucurbitaceae) (Rammal et al., 2009), Anacardium occidentale (Anacardiaceae) (Jaiswal et al., 2016), Anabasis articulata (Chenopodiaceae) (Kambouche et al., 2011) sur la glycémie des rats diabétiques. En effet, Rammal et al. (2009) ont démontré dans leurs études que l'extrait aqueux des fruits frais de Momordica charantia L., plante antidiabétique (Laleye et al., 2015) entraine une diminution de la glycémie chez les souris rendues diabétiques par l'alloxane à la dose de $200 \mathrm{mg} / \mathrm{kg}$ p.c. Ce constat a été également fait par Jaiswal et al. (2016), sur des rats rendus diabétiques par la n- streptozotocine en présence d'extrait éthanolique de feuilles de Anacardium occidentale. Enfin Kambouche et al. (2011) en étudiant l'extrait butanolique du $\beta$-sitoglucoside contenu dans Anabasis articulata sur le diabète expérimental induit par le glucose chez des souris, ont conclu que cet extrait baisse la glycémie. Nos résultats sont également semblables à ceux de Nyunaï et al. (2015) qui ont démontré dans leurs travaux, l'effet antihyperglycémiant de l'extrait aqueux de Ageratum conyzoïdes sur des rats rendus diabétiques par la streptozotocine. Atawodi et al. (2017) quant à eux ont observé cet effet avec l'extrait methanolique chez des rats rendus diabétiques par la streptozotocine. L'étude de Raphia gentiliana (Arecaceae) (Masunda et al., 2014) sur la réduction de l'hyperglycémie provoquée chez les souris et de l'extrait de l'écorce de tige de Anthrocleista djalonesis (Gentianaceae) (Ehoulé et al., 2016) sur la glycémie chez les lapins, ont également données des résultats semblables. Une étude ethnopharmacologique menée par Agbodjento et al. (2018) a montré que Gardenia ternifolia (Rubiaceae) est une plante utilisée dans la pharmacopée traditionnelle pour traiter le diabète.

Le Glibenclamide à $10 \mathrm{mg} / \mathrm{kg}$ p.c. a entrainé une chute de la glycémie chez les rats diabétiques mais cette chute est plus prononcée que celle de notre extrait. En effet, le Glibenclamide, substance hypoglycémiante de référence utilisée dans notre étude, réduit la glycémie en stimulant la sécrétion d'insuline par les cellules bêta du pancréas. En effet, le glibenclamide se fixe sur ses récepteurs à la surface de la membrane des cellules bêta pancréatiques et entraine une dépolarisation de cette membrane suivie de l'ouverture des canaux calciques calcium dépendant, ce qui entraine une entrée de calcium dans la cellule. Cette entrée de calcium aura pour conséquence, la libération de l'insuline qui va induire une baisse de la glycémie (Serrano et al., 2006). L'extrait aqueux de Ageratum conyzoïdes pourrait agir selon le même mécanisme d'action ou par d'autres mécanismes, pour réduire la glycémie des rats diabétiques.

Les paramètres hématologiques du lot de rats traités à $5000 \mathrm{mg} / \mathrm{kg}$ p.c. ne varient pas significativement $(p>0,05)$ de ceux des rats témoins normo-glycémiques. Par contre les taux de globules rouges, du volume globulaire moyen et d'hématocrites des rats témoins diabétiques baissent significativement $(p$ $<0,05)$ et plus significativement $(p<0,01)$ pour le taux d'hémoglobines par rapport à ceux des rats témoins normo-glycémiques. Le taux de globule blanc subit une augmentation significative $(\mathrm{p}<0,05)$ chez les rats témoins diabétiques par rapport aux rats témoins normo-glycémiques. Ces résultats sont semblables à ceux produits par les extraits éthanoliques des feuilles de Nauclea latifolia (Rubiaceae) chez les rats rendus diabétiques par l'alloxane (Edet et al., 2013) et de Ceiba pentandra (Bombacaceae) (Hadiza et al., 2016).

La chute de ces différents paramètres chez les rats témoins diabétiques pourrait être le résultat d'une anémie ou le début du processus de glycosylation observé chez les diabétiques (Rao et al., 2003). En effet une variation des paramètres hématologiques chez 
un sujet est synonyme d'une anomalie dans le fonctionnement, la morphologie ou le métabolisme des érythrocytes, des leucocytes et des plaquettes (Comazzi et al., 2004). La non variation des paramètres hématologiques des rats traités par rapport aux rats témoins normo-glycémiques pourrait signifier que notre extrait n'affecterait pas les paramètres hématologiques des rats diabétiques traités, mais agirait plutôt comme un régulateur de ces paramètres.

\section{Conclusion}

Dans le but de justifier l'utilisation de Ageratum conyzoïdes dans le traitement traditionnel du diabète ; nous avons étudié les effets de l'extrait aqueux d'Ageratum conyzoïdes sur des animaux rendus diabétiques par pancréatectomie partielle. Cet extrait entraine à la dose de $5000 \mathrm{mg} / \mathrm{kg}$ p.c. une baisse significative de la glycémie chez ces animaux. L'EAqAc permettrait une régulation des paramètres hématologiques des rats diabétiques traités. En effet, les paramètres hématologiques des rats diabétiques traités ne varient pas de ceux des rats normo-glycémiques à la fin de l'expérimentation comparativement aux témoins diabétiques. Il serait intéressant d'étudier les effets de cet extrait aqueux sur le foie les reins et le cœur pour une meilleure utilisation.

\section{CONFLIT D'INTERETS}

Les auteurs déclarent qu'ils n'ont aucun conflit d'intérêts.

\section{CONTRIBUTIONS DES AUTEURS}

Les auteurs ont contribué à égale valeur à la conception de l'étude, à la collecte des valeurs ainsi qu'à l'analyse des données et à la rédaction du manuscrit.

\section{REMERCIEMENTS}

Nous remercions le professeur Guédé Zirihi du laboratoire de Botanique de l'Université Félix Houphouët BoignyAbidjan, pour l'identification de la plante et le Bureau Ivoirien pour la Représentation et le Conseil Vétérinaire (BIRCOVET), pour l'assistance technique lors de la réalisation de la pancréatectomie partielle.

\section{REFERENCES}

Agbodjento E, Klotoé JR, Dramane G, Dougnon TV, Ategbo JM. 2018. Gardenia ternifolia Schumach. Et Thonn.: revue sur les aspects ethnobotanique, ethnopharmacologique, phytochimique et toxicologique. Int. J. Biol. Chem. Sci., 12(6): 2922-2932. DOI: https://dx.doi.org/10.4314/ijbcs.v12i6.34

Atawodi SE, Oluwafemi AA, Humphrey CN. 2017. Antihyperglycaemic and hypolipidemic effet of menthanol extracts of Ageratum conyzoides L (Asteraceae) in normal and diabetic rats. Trop. J. Pharm. Res, 16(5): 989-996. DOI: http//dx.doi.org/10.4314/tjpr.v16i5.4

Azzi R, Djaziri R, Lahafa F, Scckal FZ, Bennehdi H, Belkacem N. 2012. Ethnopharmacological survey of medicinal plants used in the traditional treatment of diabetes mellitus in the North Western and South Western Algeria. J. Med. plants Res, 6: 20412050.

DOI: http//dx.doi.org/10.5897/JMPR11.1796

Comazzi S, Spagnolo V, Bonfanti U. 2004. Erythrocyte changes in canine diabetes mellitus: in vitro effects of hyperglycaemia and ketoacidosis. Comp. Clin. Pathol., 12: 199-205. DOI: http//dx.doi.org/10.1007/s00580-0040502-x_Edet AE, Patrick EE, Eseyin AO. 2013. Hematological parameters of alloxan-induced diabetic rats treated with ethanol extracts and fractions of Nauclea lafiloia leaf. Eur. Sci. J, 9(27): 10-203. DOI: http//dx.doi.org/10.19044/esj.2013.v9n2 $7 \mathrm{p} \% 25 \mathrm{p}$

Kroa E, Doh SK, Soro YN, Yohou KS, Koulaï OJJD, Gbogbo M, N'guessan K, Aka J, Kouassi D. 2016. Effets de l'extrait aqueux de l'écorce de tige de Anthocleista djalonensis A. Chev (Gentianaceae) sur la glycémie des lapins. Int. J. Biol. Chem. Sci., 10(2) : 552-558. DOI : http://dx.doi.org/10.4314/ijbcs.v10i2.9

FID 2014. Atlas du diabète de la FID. $6^{\mathrm{e}}$ édition. 
Fleurentin J. 2012. L'ethnopharmacologie au service de la thérapeutique: sources et méthodes. Hegel, 2: 12-8. DOI : http//dx.doi.org/10.4267/2042/47400

Gbekley EH, Karou DS, Gnoula C, Agbodeka $\mathrm{K}$, Anani K, Tchacondo T, Agbonon A, Batawila K, Simpore J. 2015. Étude ethnobotanique des plantes utilisées dans le traitement du diabète dans la médecine traditionnelle de la région Maritime du Togo. Pan Afr Med j., 20: 437. DOI: http//dx.doi.org/10.11604/pamj.2015.20. 437.5660

Hadiza LM, Adamu YK, Musa BB, Abdullah M, Abubakar SA, Abdulrazaq TU, Usman UA. 2016. Acute oral toxicity study of ethanol extract of Ceiba pentandra as glucose lowering agent in diabetic rats. J Acute Dis, 5(3): 237-243 DOI:

http//dx.doi.org/10.1016/j.joad.2016.03.0 12

Jaiswal YS, Tatke PA, Gabhe SY, Vaidya AB. 2016. Antidiabetic activity of extracts of Anacardium occidentale Linn. Leaves on n-streptozotocin diabetic rats. J Tradit Complement Med, 7(4): 421-427. DOI: https//dx.doi.org/10.1016/j.tcme.2016.11 007

Kambouche N, Merah B, Derdour A, Bellahouel S, Younos C, Soulimani R. 2011. Activité antihyperglycémiante d'un stérol $\beta$-sitoglucoside isolé de la plante Anabasis articulata (Forssk) Moq. Phytothérapie, 9: 2-6. DOI: https//dx.doi.org/10.1007/s10298-0100603-4

Laleye OAF, Ahissou H, Olounlade AP, Azando EVB, Laleye A. 2015. Etude bibliographique de trois plantes antidiabétiques de la flore béninoise: Khaya senegalensis (Desr) A. Juss (Meliaceae), Momordica charantia Linn (Curcubiaceae) et Moringa oleifera Lam (Moringaceae). Int. J. Biol. Chem. Sci., 9(5): 2682-2700. DOI : http://dx.doi.org/10.4314/ijbcs.v9i5.38

Masunda TA, Mbala MB, Kayembe SJ, Longoma BF, Ngbolua KN, Tshibangu
DST, Mpiana PT. 2014. Activité antihyperglycémique et antiradicalaire des extraits de fruits de Raphia gentiliana De Wild. (Arecaceae). Int. J. Biol. Chem. Sci., 8(6): 2441-2451. DOI : http://dx.doi.org/10.4314/ijbcs.v8i6.7

Nyunaï N, Abdennebi EH, Bickil J, Manguelle-Dicoum MA. 2015. Subacute antidiabetic properties of Ageratum conyzoides leaves in diabetic rats. Int $J$ Pharm Sci \& Res, 6(4): 1378-1387. DOI : https//dx.doi.org/10.13040/ijpsr.09758232.6(4)

Okigbo RN, Omodamiro OD. 2006. Antimicrobial effect of leaf extract of Pigeon pea (Cajanus cajan (L) Mill sp) on some human pathogen. J Herbs Spices Med Plants, 12: 117-27. DOI: https//dx.doi.org/10.1300/j044v12n0_11

O.M.S. 2016. Organisation Mondiale de la Santé. Profils des pays pour le diabète.

Rammal H, Bouayed J, Desor F, Younos C, Soulimani R. 2009. Validation et contribution à l'étude de l'effet anthyperglycémique d'une plante médicinale, le Momordica charantia L. Phytotherapie, 7: 191-196. DOI: https//dx.doi.org/10.1007/s10298-0395-6

Rao G, Kamath U, Raghothama C, Pradeep KS, Rao P. 2003. Maternal and fetal indicator of oxidative stress in various obstetric complications. Ind $J$ Clin Biochem., 18: 6-80. DOI: https//dx.doi.org/10.1007/BF02867371

Saba AB, Oyagbemi AA, Azeez OI. 2010. Antidiabetic and haematinic effects of Parquetina nigrescens on alloxan induced type-1 diabetes and normocytic normochromic anaemia in Wistar rats. Afri Health Sci., 10(3): 276-282.

Serrano XM, Payares G, Mendoza AL. 2006. Glibenclamide, a Blocker of KATP Channels, Shows Antileishmanial Activity in Experimental Murine Cutaneous Leishmaniasis. Antimicrob. Ag. Chemother., 50(12): 4214-42116. DOI: $\quad 10.1128 /$ AAC.00617-06. 